\title{
Reflection symmetry and quantized Hall resistivity near the quantum Hall transition
}

\author{
D. N. Sheng and Z. Y. Weng \\ Texas Center for Superconductivity, University of Houston, Houston, Texas 77204-5506* \\ and National Center for Theoretical Science, P.O. Box 2-131, Hsinchu, Taiwan 300, Republic of China
}

(Received 11 December 1998)

\begin{abstract}
We present direct numerical evidence for reflection symmetry of longitudinal resistivity $\rho_{x x}$ and quantized Hall resistivity $\rho_{x y}$ near the transition between the $\nu=1$ quantum Hall state and insulator, in accord with recent experiments. Our results show that a universal scaling behavior of conductances, $\sigma_{x x}$ and $\sigma_{x y}$, in the transition regime decide the reflection symmetry of $\rho_{x x}$ and quantization of $\rho_{x y}$, independent of particle-hole symmetry. We also find that in the insulating phase away from the transition region $\rho_{x y}$ deviates from the quantization and diverges with $\rho_{x x}$. [S0163-1829(99)50312-3]
\end{abstract}

A reflection symmetry has been recently revealed ${ }^{1,2}$ in the transition between the quantum Hall $(\mathrm{QH})$ states and the high-magnetic-field insulator: e.g., the longitudinal resistivity in the insulator and its inverse in the neighboring $\nu=1$ $\mathrm{QH}$ state satisfies a relation

$$
\rho_{x x}\left(\Delta n_{\nu}\right)=1 / \rho_{x x}\left(-\Delta n_{\nu}\right),
$$

where $\Delta n_{\nu}$ is the Landau level (LL) filling number $n_{\nu}$ measured from the critical point $n_{\nu c}$. At the same time, the Hall resistivity remains well quantized, i.e.,

$$
\rho_{x y}=h / e^{2}
$$

over the whole transition regime. Similar symmetry also holds at $\nu=1 / 3 \rightarrow 0$ transition. ${ }^{1}$ Theoretically, such a reflection symmetry has been conjectured as due to an underlying charge-flux duality ${ }^{3}$ in the composite boson description ${ }^{4,5}$ of the QH transitions, which is equivalent to a particle-hole symmetry in the composite fermion description. ${ }^{6}$ Indeed, at $\nu=1 \rightarrow 0$ transition, one can easily understand Eq. (1) if a particle-hole symmetry in the fermion description is assumed in the lowest Landau level (LLL), and with using Eq. (2). But in a general situation, disorders do not necessarily retain the particle-hole symmetry of the LLL in a microscopic Hamiltonian of noninteracting fermions in the presence of a magnetic field. It is not clear theoretically whether the reflection symmetry can still remain there, even though experiments $^{1,2}$ have clearly shown that the critical point floats away from the LLL center without affecting the reflection symmetry.

The quantization of Hall resistivity (2) beyond the $\mathrm{QH}$ plateau regime is also nontrivial. A quantized Hall insulator has been discussed based on a phase-incoherent network model, ${ }^{7-9}$ and it has been also found ${ }^{8}$ that $\rho_{x y}$ may become divergent if the quantum interference is taken into account. Whether the quantization of $\rho_{x y}$ in the transition region is a classical behavior due to the absence of quantum interference at a finite temperature as discussed in those approaches or it is a universal behavior related to the property of quantum critical point ${ }^{10-12}$ of the $\mathrm{QH}$ state to insulator transition needs an independent clarification.

In this paper, we present direct numerical evidence for the reflection symmetry (1) in the $\nu=1 \rightarrow 0$ transition. A quan- tized Hall resistivity in the transition region is also obtained in accord with Eq. (2). In particular, all these results persist even when disorders are strong enough such that Landau level mixing becomes important. It suggests that the underlying particle-hole symmetry in the LLL is not crucial to the reflection symmetry as well as the quantization of the Hall resistance. These properties are found to be related to a universal scaling of conductances near the transition. The scaling functions of $\sigma_{x x}^{2}$ and $\sigma_{x y}^{*}\left(1-\sigma_{x y}\right)$ (in units of $\left.e^{2} / h\right)$ are found to be equal to each other as a universal curve that is symmetric with regard to the critical point, independent of sample sizes and disorder strengths. Furthermore, beyond the transition regime we find that $\rho_{x y}$ starts to deviate from the quantized value and eventually diverges as $\rho_{x x} \rightarrow \infty$ in the insulating regime. To test the robustness of the reflection symmetry, we also study the case where the higher QH plateaus $(\nu>1)$ are already destroyed at strong disorders, with only $\nu=1 \mathrm{QH}$ plateau left. This situation has been realized in a tight-binding lattice model ${ }^{13-15}$ and there one finds another $\nu=1 \rightarrow 0$ transition on the high filling number side. ${ }^{14}$ While the reflection symmetry and the Hall resistance quantization are still robust near the $\nu=1 \rightarrow 0$ transition on the low-filling-number side, they disappear at the transition on the high-filling side, suggesting that the existence of the $\mathrm{QH}$ plateau(s) above the Fermi energy may be crucial for both the reflection symmetry and the quantization of the Hall resistance.

We use a tight-binding Hamiltonian of noninteracting electrons:

$$
H=-\sum_{\langle i j\rangle} e^{i a_{i j}} c_{i}^{+} c_{j}+\text { H.c. }+\sum_{i} w_{i} c_{i}^{+} c_{i},
$$

where the hopping integral is taken as the unit, and $c_{i}^{+}$is a fermionic creation operator with $\langle i j\rangle$ referring to two nearest neighboring sites. A uniform magnetic flux per plaquette is given as $\phi=\Sigma_{\square} a_{i j}=2 \pi / M$, where the summation runs over four links around a plaquette. In the following we mainly focus on the $M=8$ case, while weaker fields with larger $M$ are also checked. $w_{i}$ is a random potential with strength $\left|w_{i}\right| \leqslant W / 2$, and the white noise limit is considered with no correlations among different sites for $w_{i}$. In the weak disorder limit, the mixing between Landau levels can be neglected 


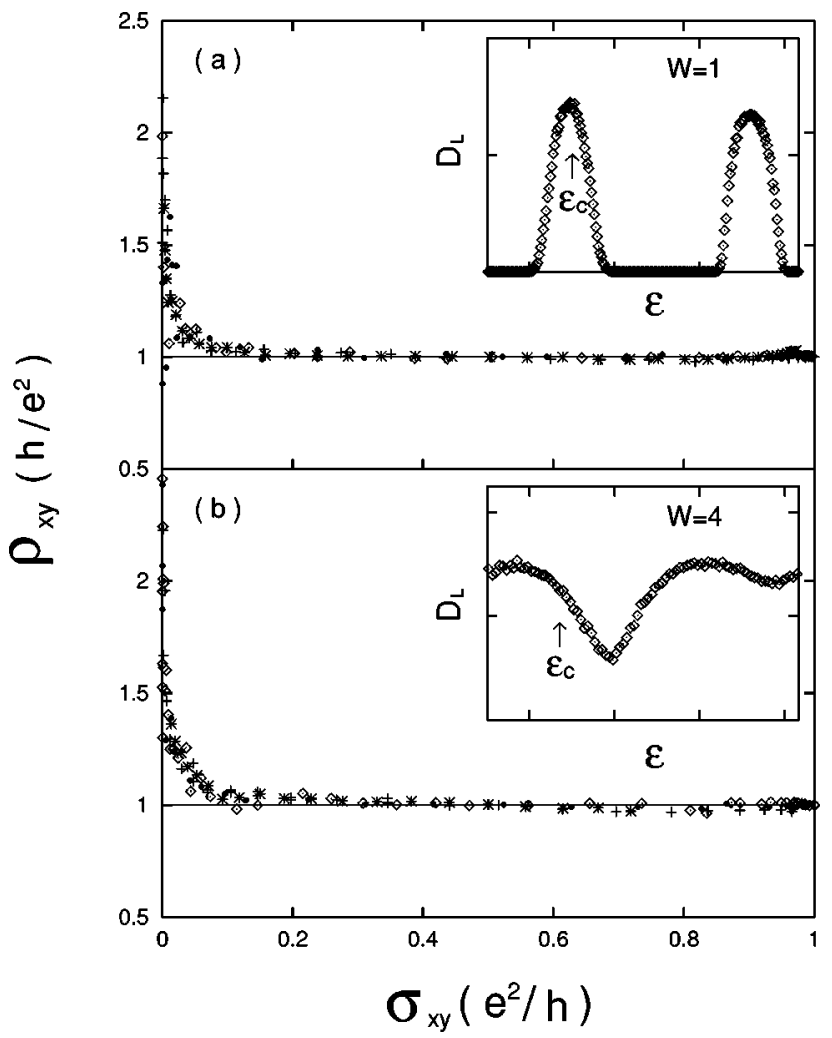

FIG. 1. The quantization of the Hall resistivity near the $\nu=1$ $\rightarrow 0$ transition: $\rho_{x y}$ versus $\sigma_{x y}$ at sample width $L=8(*) ; 16(+)$; $24(\diamond)$; and $32(\diamond)$ at disorder strength $W=1$ (a) and $W=4$ (b). The insets show the density of states $D_{L}(\epsilon) ; \epsilon_{c}$ denotes the critical point.

so the disorder effect in the LLL still approximately respects the particle-hole symmetry. With the increase of $W$, Landau levels start to mix together such that the definition of "holes" is no longer meaningful in the LLL and the particlehole symmetry is then removed. In the following, we mainly study $W=1$ and $W=4$ which represent these two limits. We also focus on the $\nu=1 \rightarrow 0$ transition that happens at the step of the Hall conductance $\sigma_{x y}$ between $e^{2} / h$ and $0 . \sigma_{x x}$ at the critical point scales onto a constant value $\sigma_{x x}=(0.50$ $\pm 0.02) e^{2} / h$ independent of disorder or magnetic field strength in agreement with earlier work. ${ }^{11,12}$ Here $\sigma_{x x}$ is calculated using the Landauer formula ${ }^{16}$ for square sample size $L \times L$ with leads and averaged over random disorder configurations to obtain a required statistical error (less than $2 \%$ ). It has been checked by us that $\sigma_{x x}$ calculated in this way agrees with the conductance calculated from the Thouless number ${ }^{17}$ as long as they are scaled to the same value at the critical point. Hall conductance $\sigma_{x y}$ is calculated by using the Kubo formula (at least 2000 configurations are taken for the largest sample size).

The quantization of the Hall resistivity is clearly shown in Figs. 1(a) and 1(b) near the $\nu=1 \rightarrow 0$ transition at $M=8$. Here $\rho_{x y}$ is plotted as a function of $\sigma_{x y}$ at different sample widths: $L=8,16,24$, and 32 , with $W=1$ and $W=4$, respectively. In such a transition regime, $\sigma_{x y}$ changes from 1 to 0 (in units of $e^{2} / h$ ), while $\rho_{x y}$ stays almost constant in the transition region at the quantized value 1 . Such a quantization remains in spite of the change of the particle-hole symmetry from $W=1$ to $W=4$ : the density of states $D_{L}(\epsilon)$ is

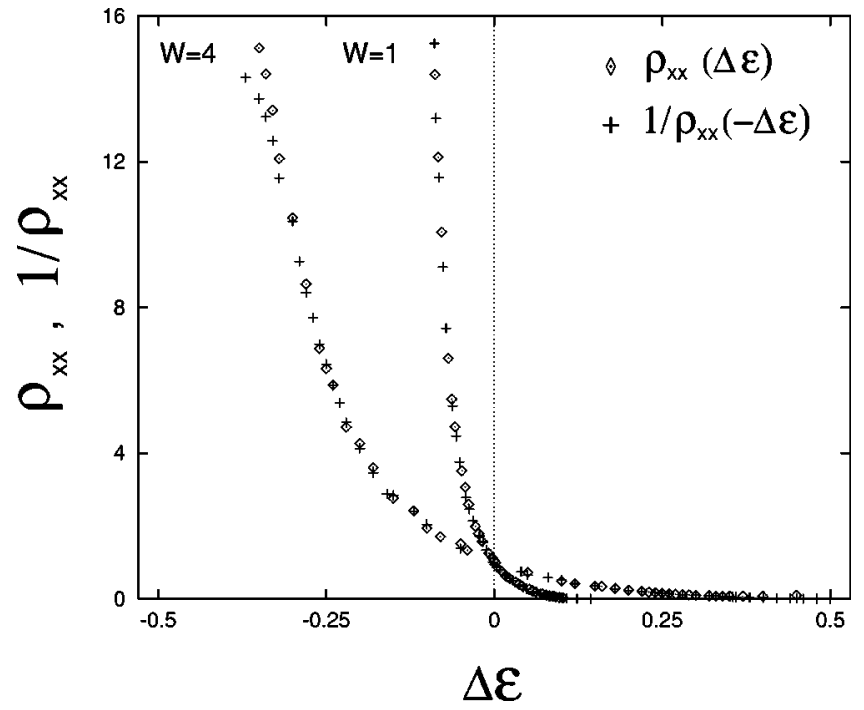

FIG. 2. The reflection symmetry of $\rho_{x x}: \rho_{x x}(\Delta \epsilon)$ and $1 / \rho_{x x}(-\Delta \epsilon)$ coincide at $W=1$ and $W=4$ with sample width $L$ $=32\left(\Delta \epsilon=\epsilon-\epsilon_{c}\right)$.

shown in the insets of Figs. 1(a) and 1(b), and in contrast to $W=1$ where the LLL is well separated from higher LL's by a gap; two lowest LL's are mixed at $W=4$ where the particle-hole symmetry is obviously absent.

The reflection symmetry of the longitudinal resistivity is shown in Fig. 2 at $W=1$ and $W=4$, respectively. Here the resistivity is plotted as a function of $\Delta \epsilon$, i.e., the Fermi energy $\epsilon$ measured from the critical point $\epsilon_{c}$. As shown in Fig. 2, $\rho_{x x}(\Delta \epsilon)$ and $1 / \rho_{x x}(-\Delta \epsilon)$ are right on top of each other, demonstrating the reflection symmetry of $\rho_{x x}$ at a sample width $L=32$. In both cases, the reflection symmetry is exhibited over a very broad region: $\rho_{x x}$ changes more than one order of magnitude in the insulating region similar to the experimental observation $^{1,2}$ (the region with reflection symmetry generally grows with the sample size $L$ in our calculation).

The quantization of the Hall resistance implies a semicircle law:

$$
\sigma_{x x}^{2}+\left(\sigma_{x y}-\frac{1}{2}\right)^{2}=\frac{1}{4},
$$

or $\sigma_{x x}^{2}=\sigma_{x y}^{*}\left(1-\sigma_{x y}\right)$, which was previously obtained based on a semiclassical treatment of the $\mathrm{QH}$ edge states. ${ }^{9}$ To make sure if this effect is an intrinsic property of a quantum critical point instead of a finite-size effect, we have checked the scaling behavior of both $\sigma_{x x}$ and $\sigma_{x y}$. It has been well established $^{11}$ that $\nu=1 \rightarrow 0$ transition should satisfy a oneparameter scaling law: both $\sigma_{x x}$ and $\sigma_{x y}$ at different sample sizes can be scaled onto a scaling curve if plotted as a function of $L / \xi$ at fixed disorder strength. Here $\xi$ is the thermodynamic localization length, $\xi=\left(\epsilon_{0} /\left|\epsilon-\epsilon_{c}\right|\right)^{\alpha}$ with $\alpha \approx 2.3$. But it was expected that the shape of the scaling curve may, more or less, depend on disorder potential. By contrast, we find that the scaling of conductances here is actually universal and the data at different $W$ 's all collapse onto a universal curve if we choose a right energy scale $\epsilon_{0}$ at different $W$ 's. For example, as shown in Fig. 3(a), $\sigma_{x x}$ at $W=1$ and $W$ $=4$ for $L=128$ and $L=160$ can be scaled onto the same 

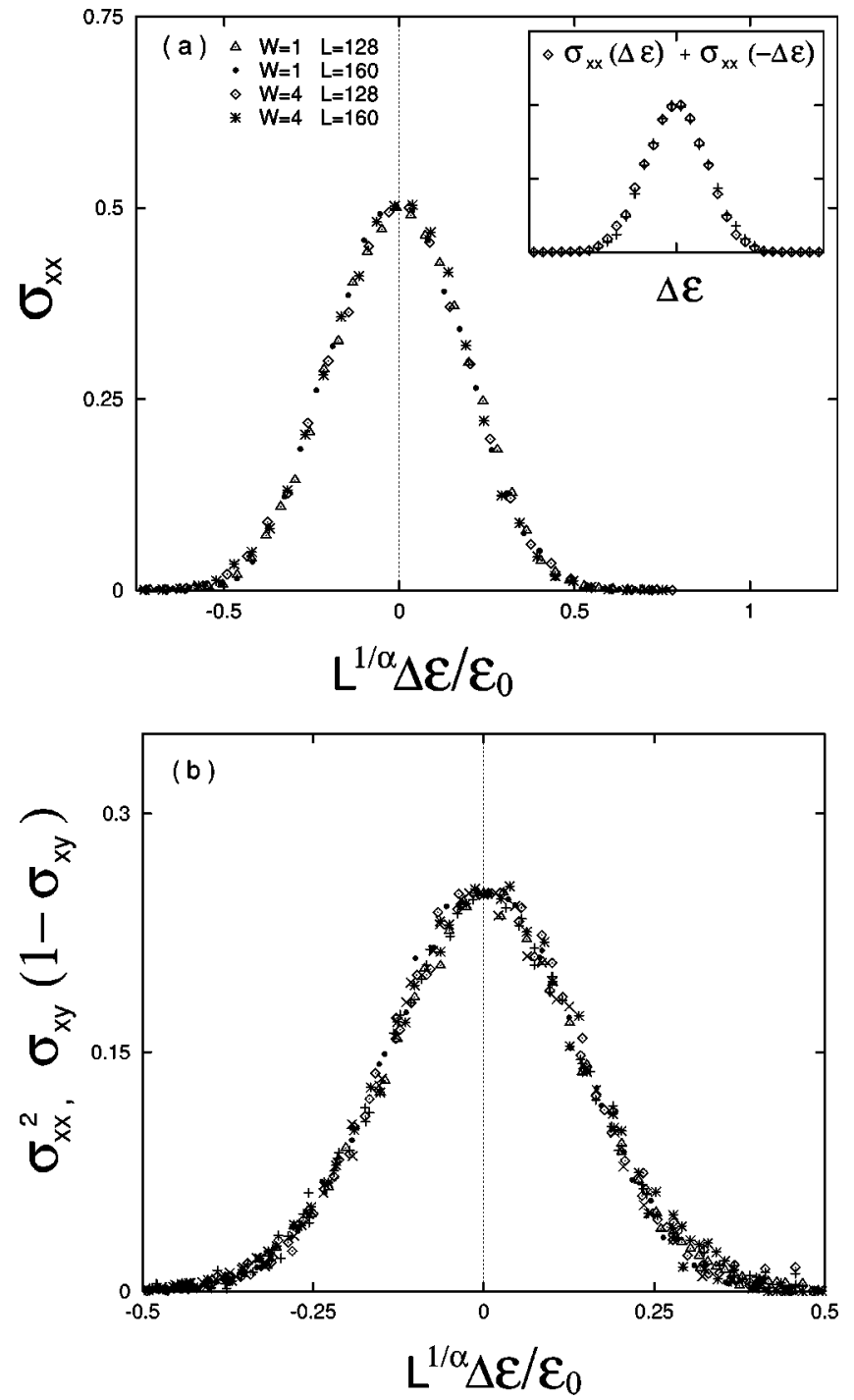

FIG. 3. (a) $\sigma_{x x}$ as a scaling function of $L^{1 / \alpha} \Delta \epsilon / \epsilon_{0}$. Here $\alpha$ $=2.3$ and $\epsilon_{0}=3.57$ at $W=4$ ( $\epsilon_{0}$ at $W=1$ is chosen as the unit). The inset shows that $\sigma_{x x}$ is a symmetric function of $\Delta \epsilon$ around the origin $(L=128$ and $W=4)$. (b) $\sigma_{x x}^{2}$ and $\sigma_{x y}^{*}\left(1-\sigma_{x y}\right)$ collapse onto a scaling function of $L^{1 / \alpha} \Delta \epsilon / \epsilon_{0}$ with $L=24,32,64,128$, and 160, and $W=1,4$.

scaling function of $L^{1 / \alpha} \Delta \epsilon / \epsilon_{0}$ by choosing $\epsilon_{0}(W=4)$ $=3.57$ with $\epsilon_{0}(W=1)$ chosen as the unit. Notice that the data shown in Fig. 3(a) are symmetric about its critical point $\epsilon_{c}$ as shown in the inset. Similar behavior is also found for $\sigma_{x y}$. In addition to the universal scaling of $\sigma_{x x}$ and $\sigma_{x y}$, we also found that the scaling curve of $\sigma_{x x}^{2}$ for sample sizes from $L=24,32,64,128$ to 160 coincides with the scaling curve of $\sigma_{x y}^{*}\left(1-\sigma_{x y}\right)$ in Fig. 3(b), independent of the disorder strength $W$. [Note that while the maximum width for $\sigma_{x x}$ calculation reaches $L=160$, the largest sample width attainable for $\sigma_{x y}$ is much smaller $(L=48)$ in the present work and in literature. $\left.{ }^{12}\right]$ This scaling relation guarantees the semicircle law (3) and the exact quantization of $\rho_{x y}$ in the whole $1 \rightarrow 0$ transition region. Thus we find that both the exact quantization of $\rho_{x y}$ and the reflection symmetry of $\rho_{x x}$ are the consequences of a universal scaling satisfied by the conductances that is independent of the details of the underlying model like the particle-hole symmetry and the lattice effect

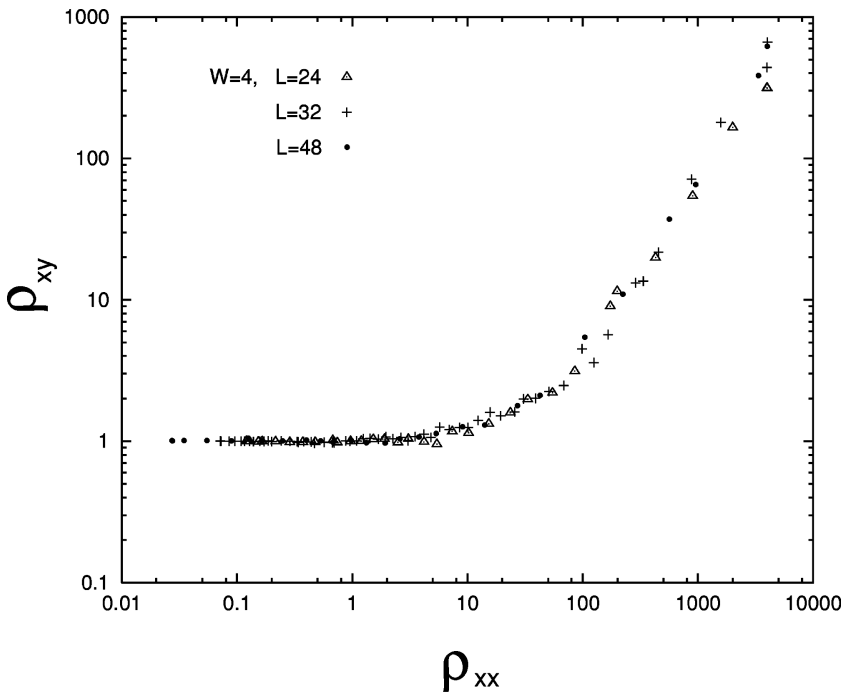

FIG. 4. $\rho_{x y}$ vs $\rho_{x x}$ in whole regime at fixed $W=4$.

(we have checked weaker magnetic fields with $M=16$ and 24 and found essentially consistent results).

It is noted that in the above numerical calculations the resistances and conductances are shown to be scaling functions with regard to the scaling variable $\xi / L$ around the critical point. Here the zero-temperature transition is driven by Fermi energy with $\xi=\left(\epsilon_{0} /\left|\epsilon-\epsilon_{c}\right|\right)^{\alpha}$. On the other hand, experiments have been done at finite temperature where the thermodynamic sample size is cut off by a finite length scale $L_{i n}$ representing the dephasing effect such that the scaling variable becomes $\xi / L_{i n}$. When a magnetic field instead of the Fermi energy is tuned in experiment, one has $\xi=\xi_{0} \mid B$ $-\left.B_{c}\right|^{-\alpha} \propto\left|n_{\nu}-n_{\nu c}\right|^{-\alpha}$. Thus, by using such a scaling variable the reflection symmetry found in our numerical calculations is related to the observed one in experiments as given in Eq. (1).

We have also checked the behavior of $\rho_{x y}$ vs $\rho_{x x}$ in whole regime. As shown in Fig. 4, the quantized $\rho_{x y}$ persists from the $\mathrm{QH}$ plateau regime with $\rho_{x x}<1$ to the transition regime with $\rho_{x x}$ as large as 5 that is comparable to the experimental data. ${ }^{1,2}$ Deep into the insulating phase with $\rho_{x x} \rightarrow \infty, \rho_{x y}$ becomes divergent too. The latter behavior is consistent with the result obtained by the network model calculation with including quantum interference. ${ }^{8}$

Finally, we have considered a case in which the reflection symmetry of $\rho_{x x}$ and the quantization of $\rho_{x y}$ near the $\nu=1$ $\rightarrow 0$ transition regime disappear. It has been previously shown that in the tight-binding lattice model the higher $\mathrm{QH}$ plateaus can be destroyed first at strong disorders while the lowest plateau remains robust. ${ }^{14}$ In this case, there exists a second $\nu=1 \rightarrow 0$ transition between the $\mathrm{QH}$ state to a high filling insulator at a critical disorder $W_{c}$ for a given Fermi energy. At $W<W_{c}, \rho_{x y}$ stays quantized in the $\nu=1$ plateau regime. But beyond $W_{c}, \rho_{x y}$ starts to deviate from the quantization and continuously drop from the quantized value approaching to the classical value $\rho_{x y}=h / e^{2} n_{\nu}$, while the reflection symmetry of $\rho_{x x}$ is no longer present. Thus, the welldefined Hall plateau(s) above the Fermi energy may be a crucial condition for both $\rho_{x y}$ quantization and the reflection symmetry of $\rho_{x x}$ to occur. This second transition has been 
related $^{14}$ to the experimentally observed $\nu=1 \rightarrow 0$ transition at weak magnetic field side, and detailed properties will be discussed elsewhere.

We conclude that the reflection symmetry of resistance and the quantization of Hall resistance are intrinsically present in the $\nu=1 \rightarrow 0$ transition in a two-dimensional noninteracting electron system at strong magnetic field. Our calculations suggest that they are determined by a universal scaling of conductances which is independent of the particlehole symmetry in the LLL. The present numerical results may thus provide a simple explanation for the recent experimental measurements in which both reflection symmetry of resistance and quantized Hall resistance were observed., One of the predictions based on our numerical study is that the quantized Hall conductance only exists near the transition region with $\rho_{x x}<10\left(h / e^{2}\right)$ which is consistent with the

*Permanent address.

${ }^{1}$ D. Shahar et al., Science 274, 589 (1996); D. Shahar et al., Solid State Commun. 102, 817 (1997).

${ }^{2}$ D. Shahar et al., Phys. Rev. Lett. 79, 479 (1997); D. Shahar et al., cond-mat/9706045 (unpublished); M. Hilke et al., Nature (London) 395, 675 (1998).

${ }^{3}$ E. Shimshoni, S. L. Sondhi, and D. Shahar, Phys. Rev. B 55, 13730 (1997).

${ }^{4}$ S. Kivelson, D.-H. Lee, and S.-C. Zhang, Phys. Rev. B 46, 2223 (1992).

${ }^{5}$ X. G. Wen and Y. S. Wu, Phys. Rev. Lett. 70, 1501 (1993); L. P. Pryadko and S.-C. Zhang, Phys. Rev. B 54, 4953 (1996); E. Fradkin and S. Kivelson, Nucl. Phys. B 474, 543 (1996); L. P. Pryadko, Phys. Rev. B 56, 6810 (1997).

${ }^{6}$ J. K. Jain, Phys. Rev. Lett. 63, 199 (1989).

${ }^{7}$ E. Shimshoni and A. Auerbach, Phys. Rev. B 55, 9817 (1997).

${ }^{8}$ L. P. Pryadko and A. Auerbach, Phys. Rev. Lett. 82, 1253 (1999); L. P. Pryadko and K. Chaltikian, Phys. Rev. Lett. 80, 584 (1998).

${ }^{9}$ A. Dykhne and I. Ruzin, Phys. Rev. B 50, 2369 (1994); I. Ruzin and S. Feng, Phys. Rev. Lett. 74, 154 (1995). existing experiments. Deep into the insulating regime with $\rho_{x x} \rightarrow \infty$, however, $\rho_{x y}$ is no longer quantized and expected to diverge as well. Thus a further experimental investigation of the insulating regime at $\rho_{x x}>10\left(h / e^{2}\right)$ is encouraged in order to fully determine the relevance of the present scaling law to experiment.

The authors would like to thank L. P. Pryadko, S. Subramanian, C. S. Ting, Z. Q. Wang, X. G. Wen, X. C. Xie, and $\mathrm{K}$. Yang for stimulating and helpful discussions. We also would like to acknowledge the hospitality of the National Center for Theoretical Science in Taiwan where the present work was partially completed. The present work was supported by the State of Texas through ARP Grant No. 3652707 and Texas Center for Superconductivity at the University of Houston.

${ }^{10}$ The Quantum Hall Effect, edited by R. Prange and S. M. Girvin (Springer-Verlag, New York, 1990); A. M. M. Pruisken, Phys. Rev. Lett. 61, 1297 (1988); H. Levine et al., ibid. 51, 1915 (1983).

${ }^{11}$ B. Huckestein and B. Kramer, Phys. Rev. Lett. 64, 1437 (1990); B. Huckestein, Europhys. Lett. 20, 451 (1992); D.-H. Lee et al., Phys. Rev. Lett. 70, 4130 (1993); Z. Wang et al., ibid. 77, 4426 (1996); X. Wang et al., Phys. Rev. B 58, 3576 (1998); B. Jovanovic and Z. Wang, Phys. Rev. Lett. 81, 2767 (1998).

${ }^{12}$ Y. Huo, R. E. Hetzel, and R. N. Bhatt, Phys. Rev. Lett. 70, 481 (1993).

${ }^{13}$ D. Z. Liu et al., Phys. Rev. Lett. 76, 975 (1996); K. Yang and R. N. Bhatt, ibid. 76, 1316 (1996).

${ }^{14}$ D. N. Sheng and Z. Y. Weng, Phys. Rev. Lett. 78, 318 (1997); 80, 580 (1998).

${ }^{15}$ H. Potempa et al., Physica B 256, 591 (1998); K. Yang and R. N. Bhatt, Phys. Rev. B 59, 8144 (1999).

${ }^{16}$ D. S. Fisher and P. A. Lee, Phys. Rev. B 23, 6851 (1981); H. U. Baranger and A. D. Stone, ibid. 40, 8169 (1989).

${ }^{17}$ R. N. Bhatt and S. Subramanian, Bull. Am. Phys. Soc. 43, 119 (1998). 